\title{
Trauma vascular y depuración de lactato como predictor determinante de mortalidad. Reporte de caso
}

\author{
Carlos A. Cortés-Samacá ${ }^{*}$, Héctor J. Meléndez-Flórez², Saúl Álvarez-Robles², Sonia M. Vivas-García ${ }^{3}$ \\ y Andrea J. Castillo-Niño ${ }^{4}$ \\ ${ }^{1}$ Hospital Universitario de Santander; ${ }^{2}$ Posgrado de Anestesiología, Universidad Industrial de Santander; ${ }^{3}$ Unidad de Cuidados Postanestésicos, \\ Hospital Universitario de Santander; ${ }^{4}$ Facultad de Salud, Escuela de Medicina, Universidad Industrial de Santander. Bucaramanga, Colombia
}

\section{Resumen}

Introducción: La medición seriada del lactato sérico es fundamental para el seguimiento de la respuesta terapéutica de reanimación inicial y el control de daños en pacientes con trauma vascular, así como predictor de morbimortalidad. Caso clínico: Presentamos un paciente masculino de 25 años, quien sufre trauma por herida de cuchillo en el tórax, con lesión vascular, en quien se realizó una medición seriada de lactato sérico, control de daños y reanimación hídrica. Posteriormente se diagnosticó una lesión vascular, y fue llevado a exploración vascular de vasos subclavios más arteriotomía por hallazgo de vasoespasmo en la arteria axilar con trombo in situ y rafia de la arteria subclavia. Conclusiones: La medición seriada del lactato sérico y su rápida depuración en las primeras horas del evento predice exitosamente una baja probabilidad de morbimortalidad a pesar de las características y la gravedad del trauma.

Palabras clave: Trauma. Depuración de lactato. Lactato. Lesión vascular.

\section{Vascular trauma and lactate clearance as predictor determining mortality. Case report}

\begin{abstract}
Introduction: The serial measurement of serum lactate is essential for monitoring the therapeutic response of initial resuscitation and damage control in patients with vascular trauma, as well as a predictor of morbidity and mortality. Case report: We present a 25-year-old male patient who suffers trauma due to knife wound in the thorax with vascular injury, in whom a serial measurement of serum lactate, damage control and resuscitation was performed; subsequently, a vascular lesion was diagnosed, and vascular exploration of the subclavian vessels + arteriotomy was carried out due to the finding of vasospasm in the axillary artery with in situ thrombus and subclavian artery raffia. Conclusions: The serial measurement of serum lactate and its rapid clearance in the first hours of the event successfully predicts a low probability of morbidity and mortality despite the characteristics and severity of the trauma.
\end{abstract}

Key words: Trauma. Clearance lactate. Lactate. Vascular injury.

Correspondencia:

Carlos A. Cortés-Samacá

E-mail: andres1236@ hotmail.com
Fecha de recepción: 02-01-2018

Fecha de aceptación: 07-01-2019

DOI: 10.24875/RAA. 19000003
Disponible en internet: 05-02-2019 Rev Argent Anestesiol. 2018;76(2):99-103 www.revistaargentinaanestesiologia.com 0370-7792 @ 2019 Federación Argentina de Asociaciones, Anestesia, Analgesia y Reanimación. Publicado por Permanyer México SA de CV. Este es un artículo Open Access bajo la licencia CC BY-NC-ND (http://creativecommons.org/licenses/by-nc-nd/4.0/). 


\section{Introducción}

El lactato sérico, como medida de la perfusión tisular ${ }^{1}$, se ha convertido desde mediados de la década de los noventa en un parámetro fundamental para el seguimiento de la respuesta terapéutica a la reanimación inicial y control de daños, así como predictor de mortalidad $^{2}$. A diferencia de los pacientes con sepsis, en quienes está ampliamente demostrado que los niveles elevados de lactato sérico se relacionan directamente con la mortalidad y morbilidad, se acepta que una depuración mayor del $20 \%$ en 6 horas mejora el pronóstico en los pacientes con trauma a pesar de que no existan suficientes estudios que determinen la depuración de lactato sérico como un eje central en la reanimación en pacientes con trauma, en estado de choque de origen hipovolémico y con hipoperfusión secundaria.

\section{Caso clínico}

Con la autorización y consentimiento informado del paciente, presentamos un caso de un paciente masculino de 25 años de edad, sin antecedentes patológicos ni quirúrgicos, que llega al Hospital Universitario de Santander por herida de arma corta punzante en la región infraclavicular derecha con línea media clavicular. Al examen físico de ingreso se percibe un paciente en malas condiciones generales, con alteración de su estado de conciencia, palidez generalizada, con una presión arterial de $45 / 30 \mathrm{mmHg}$, frecuencia cardíaca de 140 latidos por minuto y frecuencia respiratoria de 26 respiraciones por minuto. A la auscultación torácica presenta ruidos cardíacos rítmicos, taquicárdicos, sin soplos, e hipoventilación de todo el campo pulmonar derecho, con evidencia de herida en la región infraclavicular derecha con línea media clavicular. Abdomen sin alteraciones, extremidades eutróficas, con pulsos conservados y disminución del llenado capilar. Al examen neurológico se encuentra somnoliento, con escala de Glasgow 9/15. Es valorado por el servicio de cirugía general, y consideran un hemotórax masivo, por lo que le administran 2 litros de lactato Ringer, sin mejora de las cifras tensionales, por lo que es llevado inmediatamente a cirugía. Al ingreso en las salas de cirugía, se realiza monitoreo invasivo, se toman gases arteriales de ingreso que documentan acidemia metabólica con hiperlactatemia grave (valor de referencia normal de lactato sérico en gases arteriales: $<2 \mathrm{mmol} / \mathrm{dl}$ ) (Tabla 1). Inicialmente se realiza un control de daños y se traslada a la Unidad de Cuidados Postanestésicos (UCPA). En el postoperatorio inmediato se observa palidez, frialdad y disminución de pulsos en el miembro superior derecho. Se considera que es un paciente con signos de lesión vascular y se realiza un angio-TAC que evidencia interrupción de flujo arterial a nivel axilar en su tercio proximal, sin fuga del medio de contraste. Es llevado a segundo tiempo quirúrgico, donde se realiza una exploración vascular de los vasos subclavios más arteriotomía por hallazgo de un vasoespasmo en la arteria axilar con trombo in situ y rafia de la arteria subclavia más inserción del catéter venoso central. El paciente es trasladado a la UCPA, donde se toman nuevamente gases arteriales y se evidencia persistencia de acidemia metabólica, con leve elevación del lactato (Tabla 1), por lo que se hace reanimación hídrica y soporte vasopresor, terapias orientadas por metas (tensión arterial, gasto urinario, llenado capilar y depuración de lactato). A las 6 horas se documenta una depuración de lactato asociado a mejora en el estado ácido-base del paciente y destete completo del soporte vasopresor. Paciente con buena evolución clínica, en postoperatorio descrito, que al día siguiente es trasladado a salas de hospitalización general, con suplencia de oxígeno por sistema de bajo flujo estable hemodinámicamente, sin soporte vasopresor, con buen gasto urinario, con toracostomia permeable con drenaje hemático escaso. Al día siguiente, en las salas generales, se hace control con gases arteriales de control sin trastorno ácido-base, adecuados índices de oxigenación y lactato dentro de límites normales (Tabla 1).

El paciente no requirió nuevas intervenciones quirúrgicas y fue dado de alta diez días después del trauma, $\sin$ secuelas.

\section{Trauma}

El trauma ha sido identificado como la segunda causa de muerte en Colombia, después de las enfermedades cardiovasculares. Fue la causa de 15,747 muertes en el 2012, en las que las lesiones por arma de fuego y las lesiones por armas cortas punzantes encabezan el listado, causando 12,285 y 2,475 muertes, respectivamente $^{3}$. Pero no solo es un problema a nivel de Colombia, la Organización Mundial de la Salud estimó que, en el año 2000, 5 millones de personas murieron en circunstancias violentas, y la mayoría de estas muertes ocurrieron en países en vías de desarrollo ${ }^{4}$.

La causa de la muerte por trauma durante las primeras horas es generalmente de origen hemorrági$\mathrm{CO}^{1,2}$, superado solo por el trauma craneoencefáli$\mathrm{co}^{5,6}$; sin embargo, el choque hipovolémico tiene la ventaja que responde a la terapéutica que se 
Tabla 1. Seguimiento de gases arteriales y lactato en el tiempo

\begin{tabular}{|c|c|c|c|c|c|c|}
\hline \multicolumn{7}{|c|}{ Gases arteriales } \\
\hline Fecha & \multicolumn{3}{|c|}{$13 / 11 / 16$} & \multicolumn{2}{|c|}{$14 / 11 / 16$} & $16 / 11 / 16$ \\
\hline Hora & $12: 27$ & $15: 18$ & $18: 17$ & $10: 02$ & 16:10 & $15: 40$ \\
\hline Temperatura & $36^{\circ} \mathrm{C}$ & $36^{\circ} \mathrm{C}$ & $37^{\circ} \mathrm{C}$ & $36^{\circ} \mathrm{C}$ & $36^{\circ} \mathrm{C}$ & $37^{\circ} \mathrm{C}$ \\
\hline $\mathrm{PH}$ & 7.135 & 7.229 & 7.326 & 7.391 & 7.444 & 7.412 \\
\hline $\mathrm{PCO}^{2}$ & 46 & 35.1 & 42 & 41.9 & 40.3 & 46.9 \\
\hline $\mathrm{PO}^{2}$ & 218 & 167 & 149 & 94.7 & 89.7 & 46.5 \\
\hline Sat0 ${ }^{2}$ & $99.30 \%$ & $99 \%$ & $98.90 \%$ & $94.70 \%$ & $97.40 \%$ & $81.90 \%$ \\
\hline $\mathrm{FIO}^{2}$ & 0.6 & 0.35 & 0.35 & 0.32 & 0.28 & 0.21 \\
\hline Bicarbonato (mmol/l) & 15.1 & & & 14.3 & 21.4 & 29.2 \\
\hline Lactato (mmol/l) & 11.2 & 8.3 & 3.2 & 2.7 & 1.3 & 1.1 \\
\hline
\end{tabular}

instaura en la mayoría de las instituciones de salud que se adhieren a las recomendaciones de reanimación inicial con cristaloides y hemoderivados, para mantener una presión arterial sistólica normal, en el marco de la estrategia Advanced Trauma Life Support (ATLS) $)^{5,7}$, lo que disminuye la morbimortalidad entre un 30 y un $40 \%$.

\section{EVALUACIÓN CLÍNICA VS. MEDICIÓN DE LACTATO EN SANGRE}

La dificultad que se ha encontrado en la evaluación y el seguimiento a las intervenciones terapéuticas en la reanimación inicial con los signos vitales, como se recomienda en $\mathrm{ATLS}^{7}$, es que se ha demostrado que tanto la presión arterial como la frecuencia cardíaca pueden llegar a ser imprecisas y tener dificultades técnicas. Además, debido a los mecanismos neurohumorales como respuesta a la hemorragia, pueden estar dentro de los parámetros normales incluso en estados avanzados de depleción de volumen intravascular, hipoperfusión e hipoxia ${ }^{7-8}$. Es por esto que ha surgido la necesidad de estandarizar pruebas más confiables para evidenciar de forma dinámica el estado de perfusión del paciente ${ }^{8}$.

Conociendo la fisiopatología del choque hemorrágico, se sabe que una caída del volumen intravascular desencadena una respuesta sistémica predecible, mediada por cambios vasculares locales y modificaciones en el sistema neuroendocrino por estimulación adrenérgica con la descrita respuesta «pelea-huida" ${ }^{9}$, con una respuesta inicial que genera vasoconstricción en órganos con aceptable tolerancia a la isquemia (músculo, grasa, piel) para preservar el flujo sanguíneo en órganos vitales, principalmente corazón y cerebro.

\section{Fisiopatología}

Si estos mecanismos anteriormente descritos son insuficientes para garantizar la homeostasis del paciente, por persistencia de la noxa causal o por reanimación insuficiente, el paciente experimenta cambios en su medio interno, instaurándose la denominada "Triada de la muerte», que consiste en acidosis, coagulopatía e hipoperfusión ${ }^{10}$. La hipoperfusión, con la reducción secundaria del oxígeno a nivel celular, lleva a un metabolismo anaeróbico a la célula, lo que hace que se acumule piruvato ante la falta de eficiencia de la enzima piruvato deshidrogenasa para convertirlo en acetil-CoA. El exceso de piruvato se convierte en lactato por reacción a través de la enzima lactato deshidrogenasa ${ }^{11}$. El lactato, con un $\mathrm{pK}_{\mathrm{a}}$ de 3.8 aproximadamente, se libera de los tejidos acompañados por un protón, y a un $\mathrm{pH}$ por encima de 6 . El ácido láctico se disocia completamente, entonces, la producción de lactato excesiva puede dar lugar a acidosis láctica con todas las complicaciones que conocemos: efecto inotrópico negativo, vasoplejía, alteración de la disociación de la hemoglobina, reducción de la efectividad de los fármacos vasopresores, disminución de la perfusión esplácnica y alteración de la tasa de filtración glomerular, entre otros ${ }^{10}$.

De esta manera, empezaron a surgir una gran cantidad de estudios desde la década de los noventa, donde se demostró que no solo el nivel elevado de lactato sérico al ingreso, sino su aclaramiento en las primeras 
24 horas $^{1,2}$, están relacionados directamente con la morbimortalidad de los pacientes traumatizados.

\section{Discusión}

Se presenta el caso de un paciente adulto joven, sin comorbilidades, quien ingresa en el Hospital Universitario de Santander con un estado de choque hipovolémico en el contexto de un trauma por herida por arma corta punzante en el tórax, con hipoperfusión, a quien se le realiza el abordaje de manejo inicial de urgencias recomendado por el ATLS, y posterior manejo de control de daños, reanimación dirigida por metas y posterior estabilización, y en segundo tiempo quirúrgico, reparación anatómica de daños. En este paciente se realizó un seguimiento del lactato sérico reportado en gases arteriales. En el primer reporte, realizado al ingreso, se encontró hiperlactatemia (lactato $11,2 \mathrm{mmol} / \mathrm{l}$ ), lo que de forma aislada y según los estudios publicados en sepsis grave extrapolados a trauma, se relaciona con el desarrollo de falla orgánica múltiple y una mortalidad tan alta como el $89 \%$, teniendo en cuenta que la determinación de lactato sérico es útil como marcador del metabolismo aerobio secundario a hipoperfusión tisular ${ }^{13}$. En trauma se ha encontrado que, aunque el monitoreo de lactato al ingreso es bastante útil para predecir desenlaces, la tendencia del monitoreo seriado es una valiosa aproximación de mortalidad hospitalaria ${ }^{8}$. Revisando la literatura publicada sobre el tema, vale la pena destacar la revisión sistemática de $\mathrm{Kruse}^{8}$, quien recopila los estudios realizados hasta el 2011, donde encuentra ocho estudios retrospectivos de moderada calidad, en los que se evidencia la utilidad de la medición y seguimiento del lactato sérico como predictor de mortalidad. También se destaca el estudio retrospectivode Shapiro ${ }^{12}$, autoridad mundial en el tema de perfusión y microcirculación, donde plantea el lactato inicial y la depuración de lactato sérico a las 6 horas como un predictor independiente de mortalidad en pacientes con trauma. En un estudio de cohorte prospectivo observacional ${ }^{11}$, Riu considera que la depuración temprana de lactato, definido por el autor como el aclaramiento a las 2 y 4 horas, es una importante variable pronóstica independiente, y este autor, al igual que otras publicaciones ${ }^{14,15}$, plantea que deben realizarse más estudios, de carácter prospectivo, para que en un futuro se pueda contar con una adecuada evidencia científica para incorporar en las guías de toma de decisiones para la reanimación guiada por metas, la depuración temprana de lactato en los pacientes de trauma.

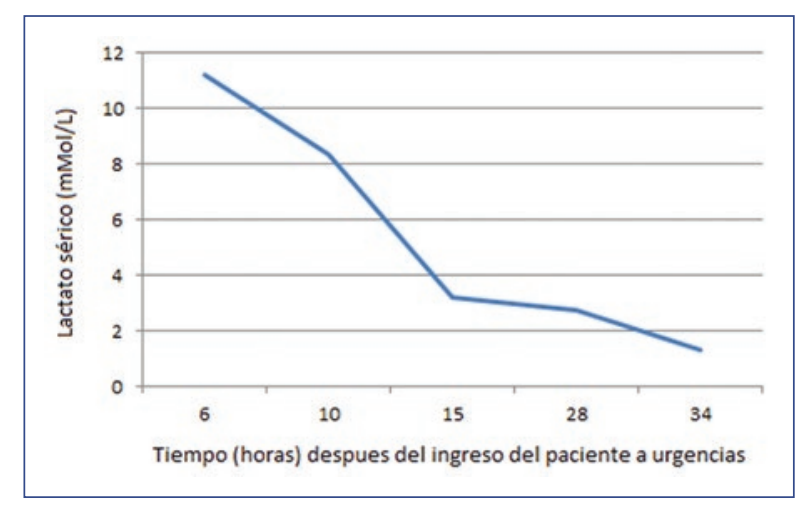

Figura 1. Depuración de lactato sérico.

En el caso analizado se evidenció una rápida depuración de lactato sérico (Fig. 1). Se encontró que el paciente depuró un $25.8 \%$ del lactato en las primeras 3 horas y $71.4 \%$ a las 6 horas, lo que predijo exitosamente una baja probabilidad de mortalidad a pesar de las características y la gravedad del trauma.

\section{Conclusión}

Si bien hay aceptación en los beneficios de la depuración temprana de los niveles de lactato sérico elevados y como variable pronóstica independiente ${ }^{16-17}$, no hay disponibles suficientes estudios de alta calidad que determinen la depuración del lactato sérico como un eje central en la reanimación en trauma ${ }^{17}$. Los distintos autores concluyen que se deben realizar estudios de carácter prospectivo para determinar si se puede extrapolar la experiencia que se tiene en $\operatorname{sepsis}^{18}$ a los pacientes traumatizados, ya que podría usarse como un marcador pronóstico sensible y de bajo costo en este tipo de pacientes. Actualmente se está desarrollando un estudio de cohorte de carácter prospectivo en nuestra institución.

\section{Responsabilidades éticas}

Protección de personas y animales. Los autores declaran que para esta investigación no se han realizado experimentos en seres humanos ni en animales.

Confidencialidad de los datos. Los autores declaran que en este artículo no aparecen datos de pacientes.

Derecho a la privacidad y consentimiento informado. Los autores declaran que en este artículo no aparecen datos de pacientes. 


\section{Bibliografía}

1. Baranov D, Neligan P. Trauma and Aggressive Homeostasis Management. Anesthesiology Clin [Internet]. 2007; 25(1):49-63.

2. Manikis P, Jankowski S, Zhang H, Kahn RJ, Vincent JL. Correlation of Serial Blood Lactate Levels to Organ Failure and Mortality After Trauma. Am J Emerg Med [Internet]. 1995;13(6):619-22.

3. Instituto Nacional de Salud [Internet]. Colombia: INS; c 2014, Boletín No. 4 [Acceso 01 de agosto de 2015]. Disponible en: http://www.ins.gov. co/lineas-de accion/ons/boletin4/interactivo/tasasM.html.

4. Krug E, Dahlberg L, Mercy J, Zwi A, Lozano R. World health report on violence and health. Geneva: World Health Organization; 2002. Disponible en: http://www.who.int/violence_injury_prevention/violence/world_report/en/summary_en.pdf?ua=1

5. Dutton RP, Mackenzie CF, Scalea TM. Hypotensive Resuscitation during Active Hemorrhage: Impact on In-Hospital Mortality. J Trauma [Internet]. 2002;52(6):1141-6.

6. Kauvar DS, Lefering R, Wade CE. Impact of Hemorrhage on Trauma Outcome: An Overview of Epidemiology, Clinical Presentations, and Therapeutic Considerations. J Trauma [Internet]. 2006:60:S3-11.

7. American College Of Surgeons [Internet]. Chicago: ACS; Capítulo 1: Evaluación y Manejo Inicial. Manual del Estudiante: Curso en Advanced Trauma Life Support ${ }^{\circledR}$. Ed 9 ${ }^{\circ}$. c 2012. P. 2-22.

8. Kruse O, Grunnet N, Barfod C. Blood lactate as a predictor for in-hospital mortality in patients admitted acutely to hospital: a systematic review. Scand J Trauma Resusc Emerg Med. 2011;28(19):74.

9. Dutton RP. Current Concepts in Hemorrhagic Shock. Anesthesiology Clin [internet]. 2007;25(1):23-34.
10. Régnier MA, Raux M, Le Manach $Y$ et al. Prognostic significance of blood lactate and lactate clearance in trauma patients. Anesthesiology [Internet]. 2012;117:1276-88.

11. Schnüriger $B$, Talving $P$, Inaba $K$ et al. Biochemical Profile and Outcomes in Trauma Patients Subjected to Open Cardiopulmonary Resuscitation: A Prospective Observational Pilot Study. World J Surg [Internet].(2012) aug; 36:1772-1778.

12. Odom SR, Howell MD, Silva GS et al. Lactate clearance as a predictor of mortality in trauma patients. J Trauma Acute Care Surg [Internet]. 2014 Mar; 76(3):902.

13. Jasso-Contreras G, González Velázquez F, Bello-Aguilar L, García Carrillo A, Muñoz-Rodríguez MR, et al. Lactato sérico como predictor de mortalidad. Rev Med Inst Mex Seguro Soc. 2015;53(3):316-21.

14. Laverde $\mathrm{CE}$, Correa $\mathrm{AF}$, Joya $\mathrm{AY}$. Lactato y déficit de bases en trauma: Valor Pronóstico. Rev Colomb Anestesiol 2014 mar;42:60-4.

15. Gunnerson K, Saul M, He S, Kellum JA. Lactate versus non-lactate metabolic acidosis: a retrospective outcome evaluation of critically ill patients. Crit Care. 2006;10(1):R22

16. Okello M, Makobore P, Wangoda R, Upoki A, Galukande M. Serum lactate as a predictor of early outcomes among trauma patients in Uganda. Int J Emerg Med [Internet]. 2014;7:20.

17. Husain FA, Martin MJ, Mullenix PS, Steele SR, Elliott DC. Serum lactate and base deficit as predictors of mortality and morbidity. Am J Surg [Internet]. 2003;185:485-91.

18. Dellinger RP, Levy MM, Rhodes A, Annane D, Gerlach H, Opal SM, et al. Surviving Sepsis Campaign: International Guidelines for Management of Severe Sepsis and Septic Shock, 2012. Intensive Care Med [Internet]. 2013;39(2):165-228. 